Daza, L., Llanes, J. y Rojas, D. (2021). La equidad en la calidad de la inserción: un análisis de los indicadōres subjetivos. Revista de Investigación Educativa, 39(2), 351-371.

DOI: http://dx.doi.org/10.6018/rie.394731

\title{
La equidad en la calidad de la inserción: un análisis de los indicadores subjetivos
}

\section{Equity in the quality of insertion: an analysis of subjective indicators}

\author{
Lidia Daza*, Juan Llanes** y Daniela Rojas** \\ *Departamento de Sociología, Universidad de Barcelona \\ ** Estudiante de Doctorado Educación y Sociedad, Universidad de Barcelona
}

\begin{abstract}
Resumen
Este artículo analiza la calidad de la inserción de los graduados catalanes en situación de ocupación tres años después de obtener el título universitario. La inserción se estudia a partir de los indicadores subjetivos del término desde la perspectiva de la equidad. Se ha indagado sobre la satisfacción del egresado con el trabajo, la valoración que hacen de las competencias adquiridas en la universidad y su utilidad en el mercado laboral, y si perciben equilibrio entre la formación y el desempeño laboral. Se ha optado por una metodología cuantitativa, analizando la Encuesta de Inserción Laboral de Graduados Universitarios de la Agència per a la Qualitat del Sistema Universitari de Catalunya (AQU) para los años 2014 y 2017. Se trata de una muestra estratificada por titulación en la que han participado 17.337 y 15.563 respectivamente. Los resultados manifiestan que las características estructurales de los titulados universitarios, especialmente ser mujer, y educativas, como tener buenas notas y haber estudiado un grado que no sea del área de Humanidades, contribuyen a configurar su percepción más positiva sobre la transición al mercado laboral en términos de indicadores subjetivos de inserción. El análisis comparado entre las dos ediciones evidencia el efecto de la crisis económica y los indicios de recuperación, muy pronunciada en el caso del área de Ing. y Arquitectura. Finalmente, Bolonia y el aprendizaje por competencias han dado sus frutos, pese a que será necesario seguir trabajando en políticas orientadas al seguimiento y acompañamiento del estudiante en la transición al trabajo.
\end{abstract}

Correspondencia: Juan Llanes, juanllanes@ub.edu. https://orcid.org/0000-0002-0059-9741. Passeig de la Vall d'hebron, 171. C.P. 08035. Barcelona. 
Palabras clave: competencias; graduados universitarios; igualdad de oportunidades; satisfacción con el trabajo.

\section{Abstract}

This article analyses the quality of job insertion for Catalan college graduates three years after obtaining a university degree. The labour integration process is studied from an equity perspective, considering subjective indicators of achievement. For this purpose, job satisfaction as well as the assessment of the skills acquired at the University and their usefulness for the labour market have been investigated. Whether students find a balance between the received training and their job performance or not has been considered too. To do so, we have selected a quantitative methodology, working with the Survey on Labour Market Insertion for University Graduates conducted by the Agency for the Quality of the University System of Catalonia (AQU), years 2014 to 2017. This is a stratified sample catalogued by university degree in which 17,337 and 15,563 students have participated respectively. Results show that some structural features of university graduates, especially being women, and other educational details such as getting good grades or studying a $B A$ degree that does not fall under humanities contribute to configuring a more positive perception of the transition to the labour market. The comparative analysis between the survey's two editions demonstrates the negative effect of the economic crisis as well as some signs of recovery, which are very pronounced in the fields of Engineering and Architecture. Finally, it is concluded that the Bologna reform and the application of competence-based learning have delivered positive results, even though it is still necessary to continue working on policies aimed at monitoring and guiding students in the process of transition from university education to the labour market.

Keywords: competences; college graduates; equal opportunities; job satisfaction.

\section{Introducción}

Incrementar la movilidad, la transferencia y el reconocimiento académico han sido procesos que, con distintos acentos, las universidades europeas han seguido desde la declaración de Bolonia (1999). Uno de los objetivos de esta transformación era contribuir con la empleabilidad de los estudiantes y su adecuación al mercado de trabajo (Martínez, González \& Rebollo, 2019). Lo cual ha significado trabajar desde un enfoque basado en competencias, en donde el estudiante es el centro articulador de la acción formativa (López, León \& Pérez, 2018; Suárez, 2016). Dada la permeabilidad del mercado y su imprevisibilidad con los cambios suscitados en el nuevo paradigma laboral es fundamental el diálogo entre universidad- trabajo para contribuir con la sostenibilidad de la sociedad.

En este marco surge el concepto de competencias, las cuales deben ser entendidas como el conjunto de conocimientos, capacidades y actitudes necesarias para la inclusión laboral (Comisión Europea, 2010). Debido a la incertidumbre del trabajo es fundamental formar tanto en competencias técnicas como transversales (Sarramona \& Santiuste, 2015). Yorke (2006) vincula el concepto al fenómeno de la empleabilidad, a partir de las características personales y profesionales del individuo que contribuyen 
a su capacidad para conseguir un empleo y mantenerlo y vivir en sociedad (Villarroya \& Ramos-Villagrasa, 2017). La empleabilidad se entiende como el conjunto de logros -habilidades, capacidades y atributos personales- que facultan a los graduados de unas condiciones óptimas para poder transitar con éxito al mercado de trabajo, conseguir un puesto laboral y ejercerlo con garantías de éxito, así como repetir el proceso tantas veces como sea necesario, ya que a lo largo de su camino profesional irán transitando por una amalgama de ocupaciones que les otorgan el título estudiado (Llanes, Figuera \& Torrado, 2017; Silva, Taveira, Bernardo \& Rodríguez, 2014). Aunque en diferentes niveles, la empleabilidad se ha transformado en uno de los principales objetivos a desarrollar por los distintos gobiernos, y que, con la idea de ser competitivos, se había impuesto en los sistemas nacionales de educación superior (Martínez et al., 2019; Pineda-Herrero, Ciraso-Cali \& Armijos-Yambay, 2018). El reto de la universidad es contribuir con la adecuación de las competencias durante el proceso formativo (Martínez \& González, 2018).

Además, es importante analizar no sólo el proceso formativo sino cómo se produce la transición de los estudiantes desde el sistema educativo al trabajo para evaluar resultados. La relación entre la educación de los jóvenes y su proceso de inserción profesional -trabajar en aquello para lo que han sido formado-incide plenamente en la utilización y aprovechamiento del capital humano, factor considerado clave en el crecimiento económico y competitividad de las naciones (González \& Martínez, 2016; Obschonka, 2014). Las dificultades de empleo a las que se enfrenta este colectivo han crecido en los últimos años, ya sea por la crisis económica en el caso español (OCDE, 2017) o por la complejidad del nuevo paradigma laboral mundial (Llanes et al., 2017), hecho que ha atraído a investigadores del campo social para su estudio.

La inserción, desde el enfoque de la carrera profesional, implica que la persona desempeñe un rol predominante en el diseño de su futuro profesional vinculado a su formación inicial. En su valoración se incluyen diversas dimensiones: objetivas, condiciones laborales -estabilidad e ingresos-, categoría profesional, y subjetivas, percepción del trabajador de las condiciones laborales. Figuera (1996) y Costa y Duarte (2011) inciden en la importancia de las variables subjetivas: contenido del trabajo, oportunidades de promoción, satisfacción global, consecución de los niveles de meta y rendimiento profesional.

La nueva realidad laboral (incierta e inestable) apela a la necesidad de estudiar otros indicadores (menos objetivos) para analizar el concepto de calidad. Siguiendo esta línea, los investigadores han estado trabajando para proponer indicadores que se ajusten más al mercado de trabajo actual. Corominas menciona la evaluación de la calidad considerando los siguientes indicadores: tipo y duración del contrato de trabajo, nivel de retribución, adecuación laboral a la titulación y satisfacción con el trabajo, en relación a la percepción con el contenido del trabajo, perspectivas de mejora y promoción y utilidad de los conocimientos en el trabajo desempeñado (Corominas, Villar, Saurina \& Fàbregas, 2012). Clasificación que, desde la comunidad científica, viene aprobada como un índice para medirla (Camelli, 2006). Donde es relevante, además, atender al bienestar del trabajador (Lent \& Brown, 2006). 
Otro de los elementos a considerar en el proceso formativo y posterior transición al trabajo, es el análisis de la desigualdad en la inserción de los titulados. Durante la última década el Espacio Europeo de Educación Superior (EEES) ha puesto especial atención al rol que juega la educación terciaria en la cohesión social y la disminución de las desigualdades, debido a que, efectivamente, los estudios universitarios son fundamentales para la ascensión social (Ariño, 2009; Berlanga, Figuera \& Pons-Farnals, 2013; Torrents \& Fachelli, 2015) y así suavizar las diferencias de clase de origen. Si bien la situación laboral de los más jóvenes se ha visto gravemente empeorada en el período de crisis con respecto a la situación laboral de la población en general, los datos muestran que disponer de una titulación universitaria protege altamente de situaciones de riesgo y vulnerabilidad social y laboral frente a las personas con estudios no universitarios; aunque existen matices por áreas de conocimientos (Fachelli \& Planas, 2014).

Especialmente, en un momento de reciente salida de una crisis económica y financiera, donde se ha producido un aumento de las desigualdades sociales, se hace necesario analizar si un título universitario tiene el mismo valor en el mercado indistintamente según las características del individuo. El género explica en muchos casos el tipo de estudio elegido por el joven (Troiano, Torrents, Sánchez-Gelabert \& Daza, 2017); encontrando así carreras más feminizadas y otras más masculinizadas. También hay múltiples evidencias de la relación intensa entre el origen social y el nivel de estudios alcanzado, aunque la relación entre nivel de estudios familiar y ocupación es más débil (Martínez García, 2017). Por ejemplo, el nivel formativo familiar aparece como un claro indicador de la disposición de recursos para los hijos destinados al estudio (Boudon, 1974; Bourdieu \& Passeron, 1990). O bien, el rendimiento se muestra como un claro configurador de expectativas, oportunidades y percepción de las propias capacidades para las elecciones educativas, en el cálculo de utilidad de la opción educativa buscando la minimización del riesgo (Erikson \& Jonsson, 1996).

La equidad educativa, por tanto, no sólo se vincula con el acceso, permanencia y titulación de los jóvenes, sino también con la constatación de igualdad de resultados en términos de inserción profesional, siendo éste un factor esencial para lograr dicha equidad. Por ende, si realmente existe equidad educativa los estudiantes de contextos socioeconómicos desfavorecidos debiesen ser igual de empleables que los demás (Formichella \& London, 2013).

Mayoritariamente el tema de la transición al trabajo y las condiciones de la ocupación se ha estudiado a partir del análisis de los llamados indicadores objetivos (Fachelli \& Navarro-Cendejas, 2015). En ellos se observa un empeoramiento generalizado de las condiciones del mercado de trabajo traducido en una mayor precarización y temporalidad de los empleos (Pineda-Herrero, Agud-Morell \& Ciraso-Calí, 2016). Acorde a lo anterior, resulta interesante indagar en factores más subjetivos que permitan conocer la calidad desde otra perspectiva del fenómeno: percepción de las condiciones laborales, ajuste formativo al título y de las competencias adquiridas, y su utilidad en el puesto laboral, focalizando el análisis en las competencias transversales. 


\section{Método}

El propósito de este estudio ha sido examinar el proceso de transición de los graduados atendiendo a la dimensión subjetiva de la calidad de la inserción (Corominas et al., 2012), desde la perspectiva de la equidad. Para ello se ha trabajado con la quinta y sexta Encuesta de Inserción Laboral de Graduados Universitarios realizada por la AQU, siguiendo una metodología cuantitativa. Dicha encuesta es el resultado de un diseño ex post facto, con una muestra representativa obtenida por procedimientos probabilísticos. Se optó por esta fuente de datos secundarios porque es un instrumento que cumple todas las garantías de fiabilidad y validez. Fue diseñado bajo el acuerdo de las instituciones universitarias catalanas y la administración en el año 2001. Al mismo tiempo que este tipo de encuestas son consideradas como un elemento privilegiado para el estudio de la transición laboral del estudiantado universitario (Delaney, 2008).

El primer objetivo ha sido abordar la satisfacción; con el contenido del trabajo, con las perspectivas de mejora, con el nivel de retribución, con la utilidad de los conocimientos para el puesto de trabajo y con el trabajo en general. En segundo lugar, examinar la valoración que hacen de la formación adquirida competencialmente y su utilidad para el trabajo que desempeñan. En ambos apartados se estudian cada una de las cuestiones anteriores contrastando las respuestas de los entrevistados en el 2014 y el 2017. A continuación, analizar la influencia que tienen variables estructurales (género, nivel formativo familiar y rendimiento), así como las diferencias que se producen según el área disciplinar de la formación cursada. Finalmente, examinar aquellas variables que han tenido un mayor impacto en la inserción.

La quinta y la sexta encuesta permiten abarcar un período temporal que recoge un ciclo de crisis económica y financiera en España con efectos directos en las condiciones económicas y educativas de las familias y en los presupuestos destinados a las políticas educativas. Y recogen un período de transformación importante en cuanto a la estructura y metodologías empleadas en la educación universitaria con la Reforma de Bolonia (1999).

\section{Población y muestra de la investigación}

La población analizada son los estudiantes universitarios catalanes titulados en 2011 y 2014, a los tres años después de haber acabado; la muestra se compone de 16.103 individuos en 2014 y 15.563 en 2017.Específicamente para este trabajo se han tomado aquellas personas que se encuentran incorporados en el mercado laboral profesional o laboralmente, representando un total del $80 \%$ (12.882 individuos) y del 84,6\% (13.166 individuos) para el 2014 y el 2017 respectivamente. A continuación (tabla 1), se muestran las principales características sociodemográficas, académicas y de inserción de los jóvenes titulados para cada oleada. 
Tabla 1

Características de los jóvenes titulados

\begin{tabular}{|c|c|c|c|}
\hline Edición encuesta & & 2014 & 2017 \\
\hline \multicolumn{4}{|l|}{ Sexo } \\
\hline & Mujer & 40,4 & 41,9 \\
\hline & Hombre & 59,6 & 58,1 \\
\hline Edad media & & 28,5 & 28,2 \\
\hline \multicolumn{4}{|c|}{ Nivel educativo familiar } \\
\hline & Estudios no universitarios & 58,3 & 52,6 \\
\hline & Estudios universitarios & 41,7 & 47,4 \\
\hline \multicolumn{4}{|l|}{ Rendimiento } \\
\hline & Aprobado & 43,7 & 31 \\
\hline & Notable, excelente, $\mathrm{MH}$ & 56,3 & 69 \\
\hline \multicolumn{4}{|l|}{ Área disciplinar } \\
\hline & Humanidades & 5,6 & 8,5 \\
\hline & Sociales & 46,7 & 46 \\
\hline & Salud & 19,1 & 18,5 \\
\hline & Ingeniería y arquitectura & 22,5 & 20,9 \\
\hline & Experimentales & 6,1 & 6,1 \\
\hline \multicolumn{4}{|l|}{ Situación laboral } \\
\hline & Trabajo & 89,1 & 92,7 \\
\hline & $\begin{array}{l}\text { No trabajo, pero he trabajado después de } \\
\text { los estudios }\end{array}$ & 10,9 & 7,3 \\
\hline & $\begin{array}{l}\text { No he trabajado nunca después de los } \\
\text { estudios }\end{array}$ & 0 & 0 \\
\hline
\end{tabular}

Nota: Elaboración propia.

\section{Instrumento, procedimiento $y$ análisis de datos}

En relación al instrumento de recogida de información utilizado por la AQU, se utiliza la encuesta telefónica como el medio más adecuado a la accesibilidad de la muestra. Del total de preguntas que recoge el cuestionario, en este artículo se trabaja con:

- Características sociodemográficas (características personales y académicas), estatus de la inserción (ocupados y desocupados).

- Escala de satisfacción con las siguientes dimensiones de la ocupación: el contenido, la perspectiva de mejora, el nivel de retribución, utilidad de los conocimientos y satisfacción general. 
- Escala de satisfacción con la formación (Alfa de Cronbach 0,896 -2014- y 0,910 -2017-) y utilidad en el mercado laboral (Alfa de Cronbach 0,866 -2014- y 0,910 -2017-) de las siguientes competencias: formación teórica, formación práctica, expresión oral, expresión escrita, trabajo en equipo, liderazgo, solución de problemas, toma de decisiones, creatividad, pensamiento crítico, gestión, informática, idiomas y habilidades de documentación.

- Ajuste entre la formación y las funciones de la persona titulada en el puesto de trabajo: variable construida siguiendo la tipología de Mañé-Vernet \& MiravetArnau (2007) a partir de las preguntas:

- ¿Qué se necesitaba para este trabajo? (1) La titulación específica (2) Sólo ser titulado (3) No hacía falta titulación universitaria

- ¿El trabajo que haces o hacías es propio de tu titulación?

- Para este trabajo, ¿crees que es necesaria la titulación?

De los cuatro perfiles de egresados que definen dichos autores, nos centramos en dos: aquellos que tienen la titulación específica necesaria para el trabajo que desempeñan (adecuadamente titulados) y los que tienen el nivel universitario requerido (aunque no la titulación específica) (adecuadamente educados).

Para abordar si una vez adquirida la titulación universitaria persisten desigualdad en las oportunidades laborales, se hace un análisis de los anteriores indicadores de acuerdo con los principales ejes de desigualdad social: sexo, nivel educativo familiar, rendimiento y área disciplinar.

Para el análisis de los datos se ha utilizado el paquete estadístico SPSSS de IBM (versión 25.0), empleando tablas de contingencia y diversas técnicas multivariantes de acuerdo con la naturaleza de la información tratada. Se utilizan pruebas no paramétricas, concretamente el test de suma de posiciones (ranks) de Wilcoxon (o U de MannWhitney) y el test $\mathrm{H}$ de Kruskal-Wallis. Finalmente, se utiliza la técnica del análisis de segmentación (tree classification) para identificar qué perfil de estudiante es el que presenta un mejor ajuste en el mercado laboral.

\section{Resultados de la investigación}

\section{Satisfacción del egresado con el trabajo}

La satisfacción con el trabajo constituye uno de los principales indicadores subjetivos de la calidad de la inserción. Los ítems que recoge el cuestionario de la AQU se valoran entre 1 (nada satisfecho) y 7 (muy satisfecho). A continuación, se expresan los resultados transformados en una escala de 1 a 10.

La puntuación más baja es un 6, referida al nivel de retribución en el 2014, y la más elevada es un 8,2 referida al contenido del trabajo en el 2017. En general, siguiendo con la comparativa entre los jóvenes encuestados en el 2014 y el 2017, los análisis muestran una mejora en las puntuaciones obtenidas para el último año, excepto en el caso de la utilidad de los conocimientos del propio trabajo, que presenta los mismos valores que para el 2014. 


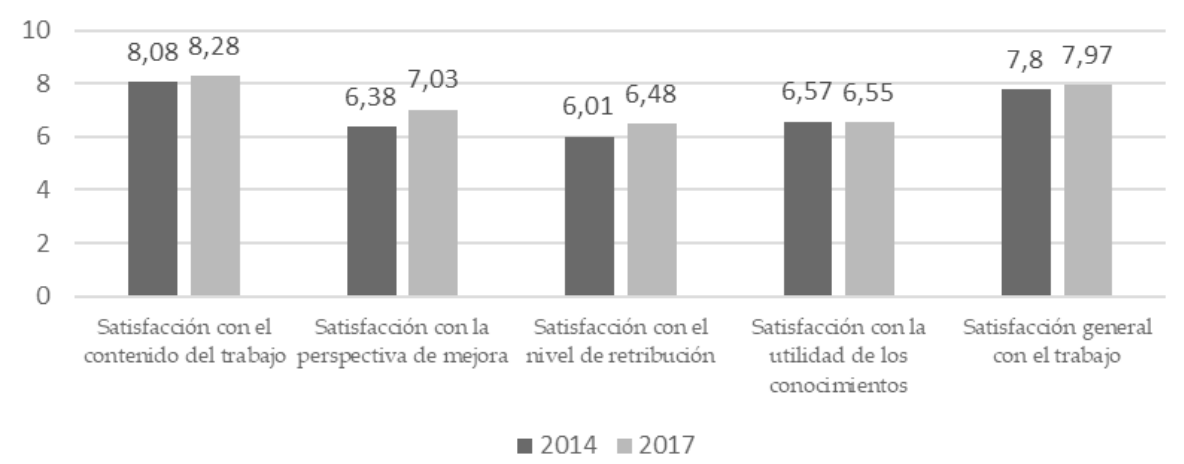

Figura 1. Valoración media de la satisfacción con la ocupación.

\section{Satisfacción según las características de los titulados}

Según el sexo, se evidencian diferencias significativas en cuatro de los cinco ítems tanto en los encuestados el 2014 como el 2017. En el caso de la edición 2014, la satisfacción con el nivel de retribución es el único que no presenta diferencia y en el 2017, la satisfacción con las perspectivas de mejora. Ahora bien, en general, las mujeres están más satisfechas que los hombres, sus medias son más elevadas en la mayoría de los ítems (entre 1 y 3 décimas más). En el caso de los hombres, puntúan más alto la satisfacción con las perspectivas de mejora en ambas ediciones. Sin embargo, a pesar de estas diferencias, ambos grupos aumentan su valoración el 2017 con respecto al año 2014.

Respecto al nivel de estudios de los padres, en general sus puntuaciones son bastante similares para las dos categorías. Sin embargo, la satisfacción con las perspectivas de mejora es mejor valorada por los encuestados con padres universitarios (edición 2014 media 6,5 v/s 6,3, edición 2017 media 7,1 v/s 7). Por el contrario, los encuestados con padres sin estudios universitarios valoran mejor la satisfacción con la utilidad del conocimiento (edición 2014 media 6,7 v/s 6,5, edición 2017 media 6,6 v/s 6,5). Las valoraciones en el 2017 son de nuevo mejores que las del 2014.

Ahora bien, al considerar el rendimiento de los estudiantes, aquellos con buenas notas (notables, excelente, $\mathrm{MH}$ ) puntúan mejor todos los ítems en comparación a los estudiantes con notas más bajas (entre 1 y 5 décimas más). Por lo que se observa, se hallan diferencias significativas en la mayoría de los ítems, únicamente la satisfacción con la perspectiva de mejora no presenta diferencia. En relación a las puntuaciones, el primer grupo puntúa mejor en todos los ítems, no obstante, siguiendo la línea de las variables anteriores para ambos grupos las valoraciones mejoran para el 2017.

\section{Satisfacción según área disciplinar}

Se evidencian diferencias significativas entre áreas. Las áreas más satisfechas son Sociales y Salud. La primera área puntúa más alto en tres de los cinco ítems (satisfacción con el contenido del trabajo, con las perspectivas de mejora y nivel de retribución) hasta con 
7 décimas por encima. El caso del área de la Salud es similar, puntuando mejor los ítems siguientes: satisfacción con el contenido del trabajo, utilidad del conocimiento y general con el trabajo. Por el contrario, el área Humanidades es la más insatisfecha, en especial, con la satisfacción con la perspectiva de mejora y nivel de retribución, con puntuaciones inferiores a 6 para la edición del 2014. Ahora bien, en general la puntuación para el 2017 mejora.

\section{Competencias adquiridas y su utilidad en el puesto de trabajo}

Otra de los indicadores examinados ha sido cómo valoran ciertas competencias los titulados una vez se ha producido el proceso de transición al mercado de trabajo. Ítems que oscilan entre el 1 'muy bajo' y el 7 'muy alto'. De nuevo expresamos los resultados en una escala de 1 a 10.

La formación recibida pasa del aprobado (figura 2). Especialmente para las competencias de trabajo en equipo, formación teórica y pensamiento crítico. Sin embargo, las que peores notas sacan son: idiomas, informática y liderazgo. De la figura también se deriva que, pese a Bolonia y la incorporación de un aprendizaje más centrado en competencias, no se puede hablar de una mejora de acuerdo a las puntuaciones de los estudiantes al comparar aquellos encuestados en el 2014 (los que estudiaron en el sistema universitario anterior) con los del 2017 (los que estudiaron por completo en el plan Bolonia).

Otra cuestión diferente es la utilidad de dicha formación en el puesto de trabajo. Este indicador aprueba (figura 3). Las competencias que consideran más útiles son también las que han valorado mejor en el apartado de formación recibida. Y la formación que adquirieron en idiomas sigue siendo la menos útil en el mercado. Pero, además, en términos comparativos, la utilidad recibe una menor puntuación para casi todas las competencias por aquéllos que estudiaron con el plan Bolonia (excepto para la formación práctica y teórica). Si bien al valorar la formación, la diferencia entre las puntuaciones de la edición del 2014 y del 2017 era escasa, en el caso de la utilidad hay mayores diferencias entre ediciones.

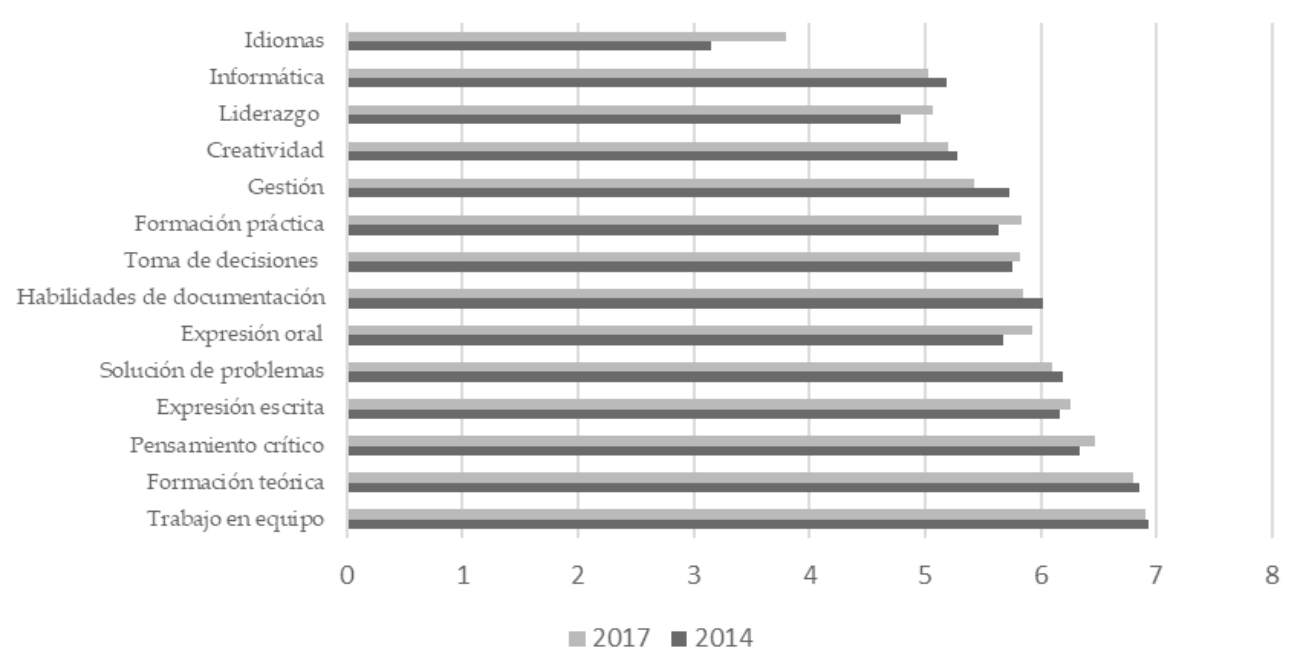

Figura 2. Valoración media de la formación competencial recibida en la universidad. 


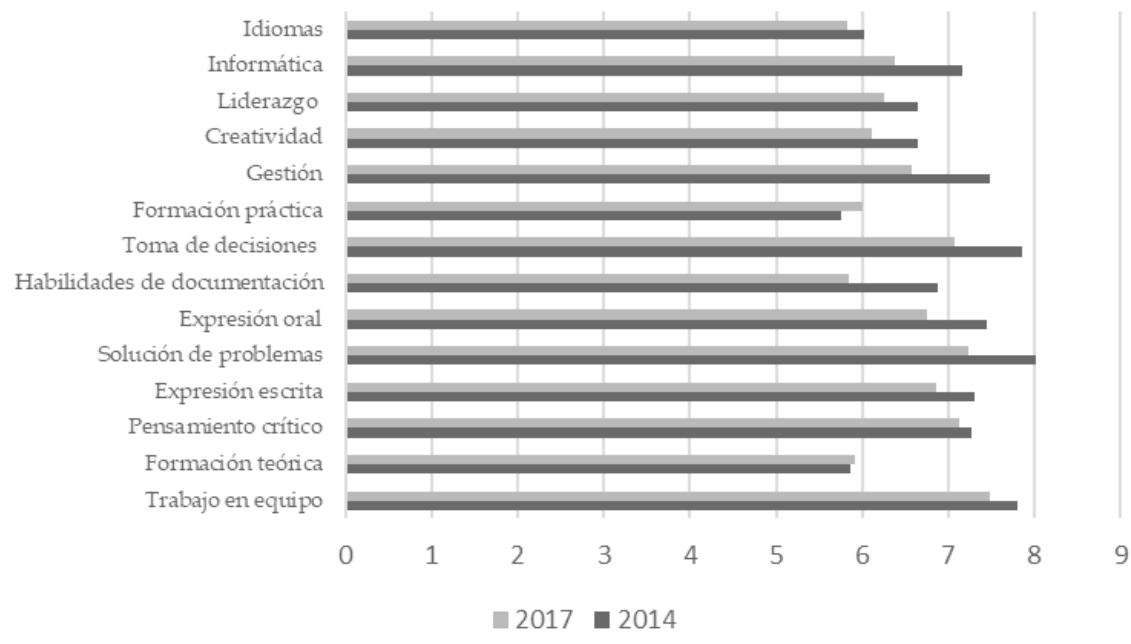

Figura 3. Valoración de la utilidad de las competencias en el mercado de trabajo.

Para hacer una mejor aproximación entre la adecuación de la formación y las demandas del mercado, se ha calculado la diferencia entre la valoración que hacen los jóvenes de cada competencia y su grado de utilidad en el puesto de trabajo. La figura 4 muestra así el déficit formativo existente para cada una de las competencias. La longitud de las barras dibuja la mayor o menor diferencia entre la valoración que hace el estudiante de la competencia y la utilidad que le asigna en el mercado. El ajuste que se produce es mayor una vez implementada la reforma universitaria que con el sistema anterior. La única competencia donde no existe déficit es en la formación teórica.

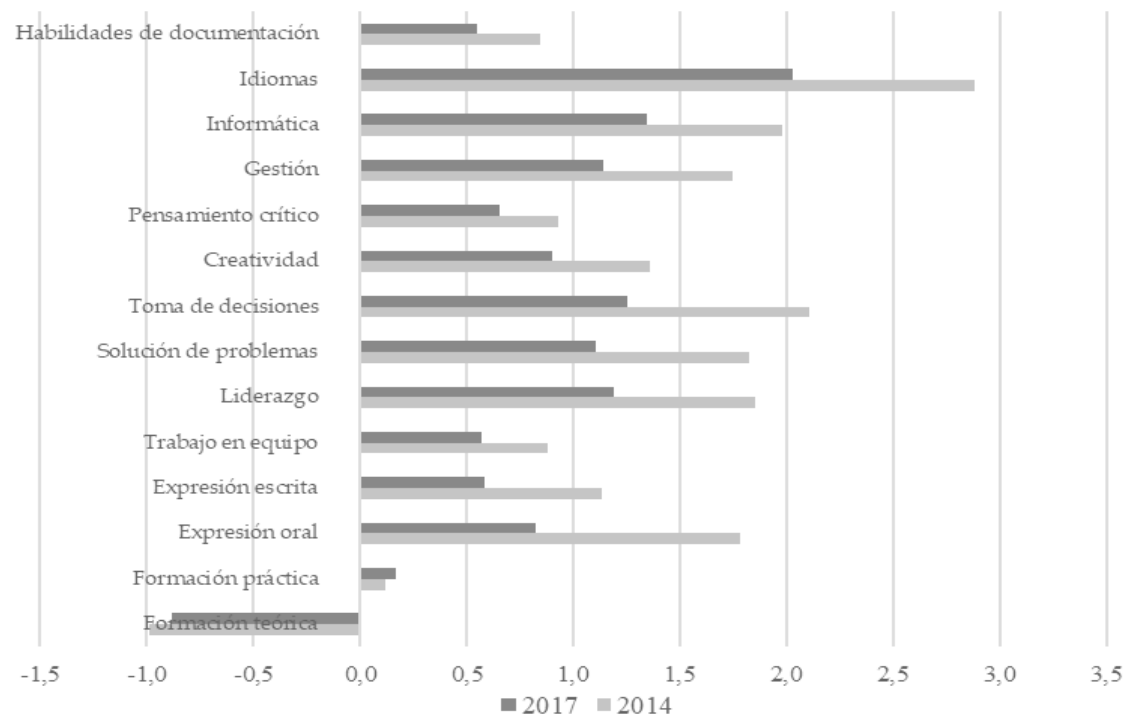

Figura 4. Déficit formativo por cada competencia. 


\section{Competencias adquiridas y su utilidad en el puesto de trabajo según las caracterís- ticas de los titulados}

Se estudian también las respuestas de los jóvenes egresados desde una perspectiva de equidad. Es decir, hasta qué punto la diferente posición en la estructura social explica una percepción diferente sobre algunos indicadores subjetivos de la calidad de la inserción. Y cómo esta distancia de percepciones puede además verse acentuada cuando las diferencias en las oportunidades laborales, económicas y educativas se acentúan.

Respecto a la valoración que hacen de las competencias adquiridas, los análisis para el 2014 manifiestan que existen claras diferencias según las características estructurales y educativas. Las mujeres, las personas con padres con estudios no universitarios, y los titulados con mejores notas son quienes mejor valoran la formación. Aunque existe alguna excepción en informática, solución de problemas o formación práctica. Para el año 2017 sucede algo parecido. No obstante, las diferencias en función del nivel educativo de los padres pierden significatividad estadística para la mayoría de las competencias.

Tabla 2

Valoración media de la formación en competencias desde la mirada de la equidad

\begin{tabular}{lrr}
\hline & 2014 & 2017 \\
\hline Hombre & 5,47 & 5,59 \\
Mujer & 5,85 & 5,88 \\
Padres con estudios universitarios & 5,60 & 5,72 \\
Padres con estudios no universitarios & 5,77 & 5,80 \\
Aprobado & 6,82 & 5,49 \\
Notable, excelente, MH & 7,16 & 5,88 \\
\hline
\end{tabular}

En relación a su utilidad, en el 2014 el género y el rendimiento del egresado también se relacionan con una diferente percepción. Las mujeres y los titulados con mejor rendimiento son quienes indican una mayor utilidad. Aunque también hay alguna excepción en competencias como el liderazgo o la informática especialmente, para las que no se producen diferencias. La influencia en este caso del nivel educativo familiar no resulta ser clara a la hora de explicar las diferentes valoraciones. Para el 2017 el nivel educativo familiar vuelve a perder peso.

Tabla 3

Utilidad media de las competencias desde la mirada de la equidad

\begin{tabular}{lcc}
\hline & 2014 & 2017 \\
\hline Hombre & 6,86 & 6,45 \\
Mujer & 7,12 & 6,67 \\
Universitarios & 7,04 & 6,61 \\
No universitarios & 7,01 & 6,54 \\
Aprobado & 5,47 & 6,33 \\
Notable, Excelente, MH & 5,85 & 6,69 \\
\hline
\end{tabular}




\section{Competencias mejor valoradas y con mayor utilidad según área disciplinar}

El análisis por áreas de conocimiento se hace más complejo, pues dependiendo del grupo, las competencias desarrolladas van a diferir ocupando las horas de formación dedicadas mayor o menor relevancia dentro del currículum. Lo cual implicará diferencias importantes en las valoraciones que reciba dicha formación por parte de los titulados (Herranz \& Muelas, 2019). Sin negar el interés que tiene su análisis desde un punto de vista de la evaluación de los programas de formación, se aleja del objetivo de este trabajo. Por ello, y por las limitaciones en la extensión del trabajo, nos centramos en el análisis de la competencia transversal que recibe una mayor puntuación, tanto por la formación recibida como por la utilidad que perciben de ella en el mercado, si ha mejorado de una edición a otra, y de qué manera esta percepción puede diferir según las características de los titulados.

En el caso de la valoración que se hace de las competencias aprendidas en la universidad, examinamos el trabajo en equipo; la que mejor puntuación recibe, siendo la competencia transversal por excelencia en cualquier estudio de grado. En ambas ediciones se obtienen puntuaciones muy parecidas. Humanidades y Experimentales son las áreas en las que se dan más décimas de diferencia entre el año 2014 y el 2017, produciéndose una mejora. Pero las áreas que mejor puntúan el trabajo en equipo como valor formativo recibido son las áreas de Sociales y Salud (figura 5).

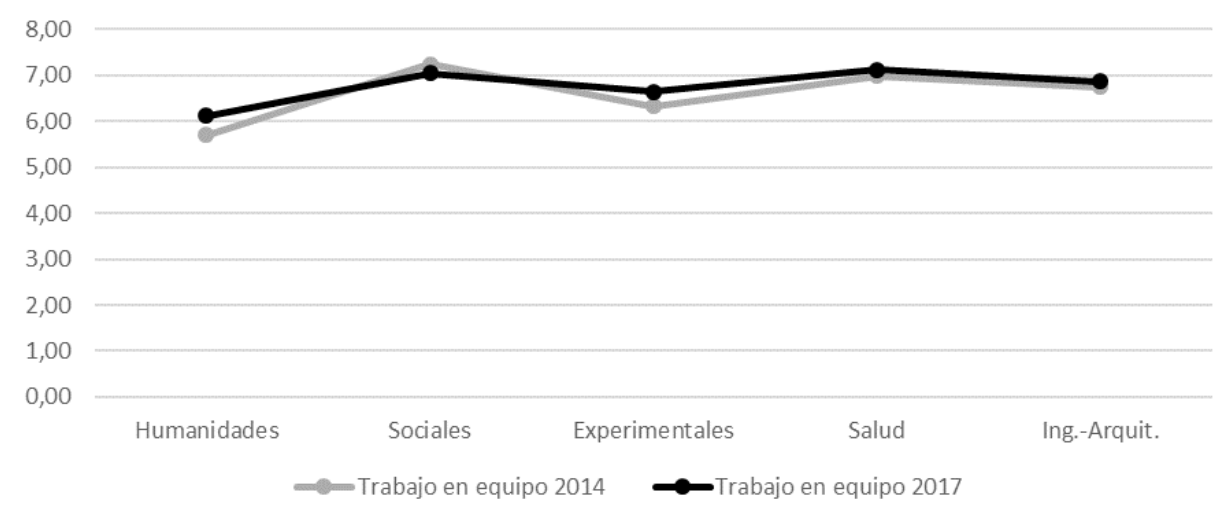

Figura 5. Valoración de la formación en trabajo en equipo según área disciplinar.

A continuación, la prueba Kruskal-Wallis permite comprobar si las diferencias en las medias entre áreas son estadísticamente significativas. Además de proporcionar el diagrama de caja, en el que se representa en qué valor se sitúa la mediana de la distribución y permite comparar el grado de heterogeneidad de la muestra en su respuesta para las diferentes áreas. 

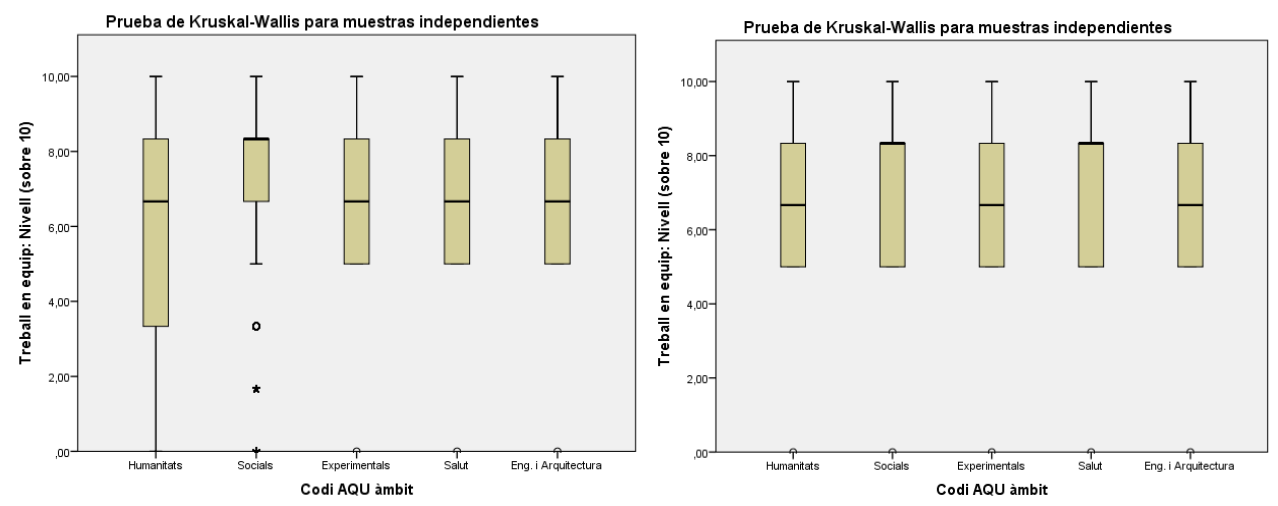

Figura 6. Diagramas de cajas para la valoración del trabajo en equipo según área disciplinar (2014, 2017).

El área de Experimentales e Ingeniería y Arquitectura mantienen un mismo patrón de distribución y sin cambios en el tiempo. Valoran el trabajo en equipo como competencia adquirida en la carrera con buena nota. En el resto de las áreas se producen cambios entre el 2014 y el 2017 en el modo cómo se distribuyen los encuestados a la hora de valorar la formación recibida en torno a dicha competencia. Los titulados en Humanidades responden de forma más homogénea en el 2017 respecto al 2014, donde se daban valoraciones más dispersas y extremas. Algo parecido sucede con el área de Sociales, que ha pasado de expresar unas opiniones muy polarizadas, a tener una opinión más consensuada y positiva. Por último, en el área de Salud lo que sucede es que aumenta el número de personas con valoraciones más elevadas.

En el caso de la utilidad la percepción de los encuestados una vez inseridos en el mercado laboral, se dan oscilaciones de un máximo de un punto. Es interesante, al hilo de lo dicho anteriormente, cómo la percepción de utilidad empeora para todas las áreas del 2014 al 2017. Claramente el área de Humanidades es donde ven menos útil dicha competencia una vez incorporados en el mercado laboral.

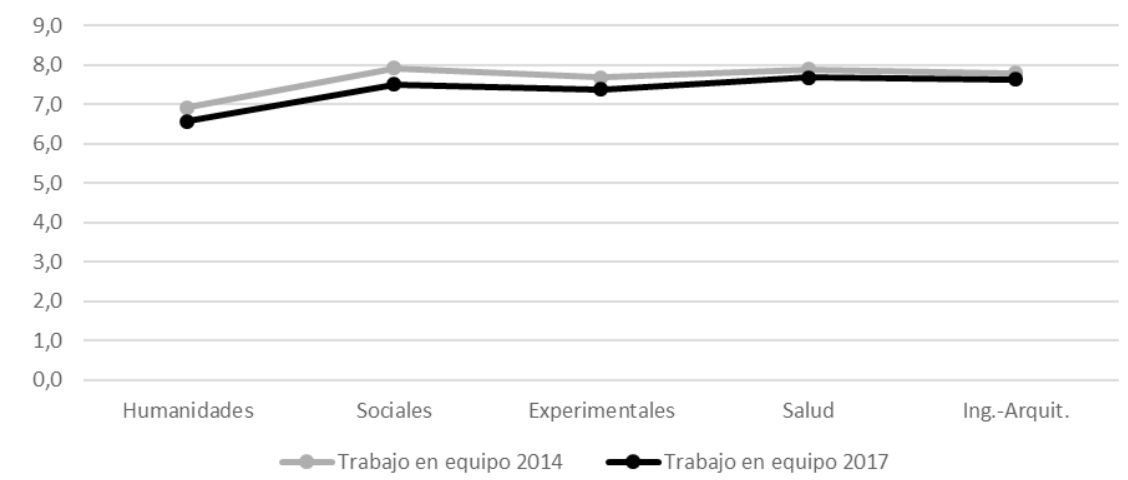

Figura 7. Valoración de la utilidad del trabajo en equipo según área disciplinar (2014). 

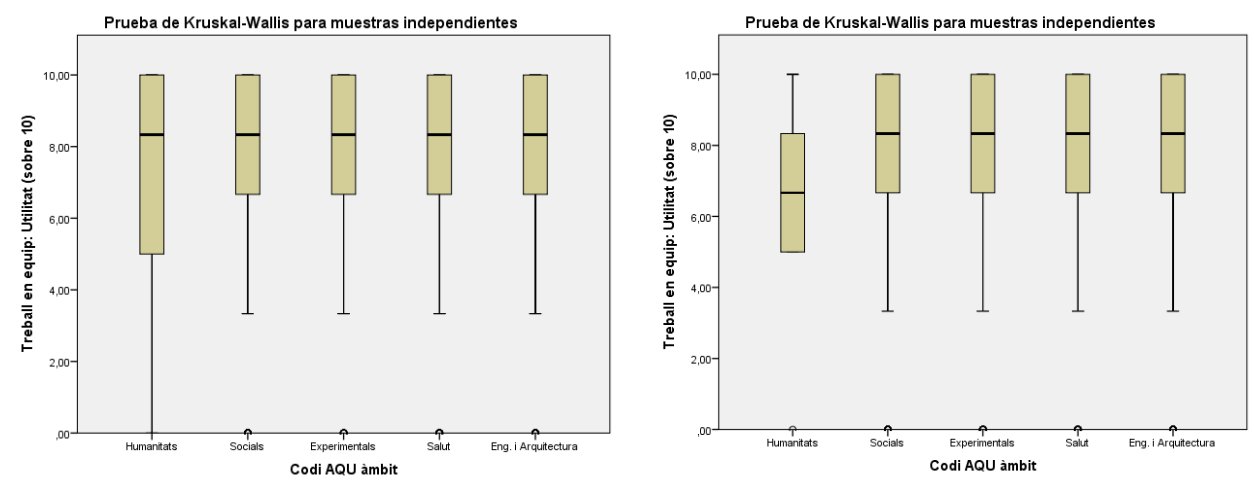

Figura 8. Diagramas de cajas para la utilidad del trabajo en equipo según área disciplinar (2014, 2017).

Respecto a la utilidad del trabajo en equipo se aprecia que el cambio fundamental que se produce entre las dos ediciones es en el área de Humanidades que, si bien para el 2014 las tres cuartas partes de la muestra de titulados daban una puntuación mayor a 5, el 25\% restante suspendía sobre la utilidad de dicha competencia. En cambio, en el 2017 las puntuaciones se desplazan hacia la parte superior de la escala, y presentan una distribución más homogénea. Para el resto de las áreas, sin cambios entre ediciones, las puntuaciones son muy positivas, aunque siempre hay un $25 \%$ de los titulados que puntúan la utilidad con valores situados entre el 3,5 y el 6,5.

\section{Ajuste en el mercado laboral}

Finalmente se analiza el nivel de ajuste del titulado entre la formación adquirida y las funciones desempeñadas en su puesto de trabajo, de acuerdo a la información recogida en la encuesta. Se ha adaptado la tipología de Mañé-Vernet \& Miravent-Arnau (2007) explicada en la descripción del instrumento.

Lo primero que debe resaltarse es que más de la mitad de los titulados trabajan de lo que estudiaron y por tanto son definidos como adecuadamente titulados. En términos de la evolución que se ha dado en los últimos años, con diferentes cambios en la estructura de los estudios y con los efectos palpables de la crisis, este dato disminuye muy ligeramente en el 2017 (65,8\%) respecto al 2014 (71,9\%) (Figura 1 y 2. Apéndice). Aunque aumenta también el colectivo de los adecuadamente educados (pasando del $28,1 \%$ al $34,2 \%$ ). Es decir que, pese a haber estudiado un grado, no acaban trabajando de esa titulación específica, pero sí desempeñando funciones universitarias.

Para acabar, mediante el análisis de segmentación (tree classification) (Escobar, 1998), exploramos cuál es el perfil del titulado con mayor representación en el grupo de los titulados con un ajuste en el mercado laboral adecuado a la titulación para las dos ediciones de la encuesta. Tanto para el año 2014 como para el 2017, el área disciplinar es el factor que mejor discrimina el colectivo con mejor ajuste en el mercado de acuerdo con su titulación $\left(\mathrm{Chi}^{2}\right.$ de Pearson=390,626, df=4, sig=0,000; $\left(\mathrm{Chi}^{2} \mathrm{de}\right.$ Pearson=308,186, 
$\mathrm{df}=4$, sig=0,000). Los que cursan titulaciones del ámbito de la Salud son quienes en mayor proporción acaban trabajando de lo que estudiaron. A continuación, se encuentra el área de Experimentales. Ingeniería y Arquitectura es el tercer ámbito con mayor ajuste en el 2017, mientras que pasó por delante el área de Sociales en el 2014. La siguiente variable discriminante en todos los casos es el sexo del encuestado en el caso del 2014 (para Humanidades, Sociales y Salud), siendo las mujeres quienes presentan una mayor adecuación a la titulación en el puesto de trabajo. Y a continuación el rendimiento indica que las mujeres de estas áreas con un rendimiento elevado son quienes en mayor proporción ocupan en el mercado laboral una posición ajustada a su titulación. Mientras que, en el 2017, adquiere mayor importancia el rendimiento en el área de Sociales, Experimentales e Ingeniería y Arquitectura, y como tercer factor discriminante se encuentra el sexo en el caso de Sociales e Ingeniería y Arquitectura.

\section{Conclusiones y discusión}

Los resultados nos han permitido aproximarnos a la dimensión subjetiva de la calidad de la inserción de los universitarios ocupados desde la perspectiva de la equidad. Los datos también permiten comparar las opiniones de la muestra de titulados en el 2014 y en el 2017, recogiendo momentos coyunturales diferenciados como comentamos anteriormente.

Diversas investigaciones plantean cómo los estudios universitarios pueden actuar como elemento de movilidad social una vez los jóvenes hacen la transición al mercado laboral. Pues a menudo el título universitario parece reducir las desigualdades sociales al analizar los indicadores objetivos de la calidad de inserción (Fachelli \& Planas, 2014). ¿Desaparecen también las diferencias estructurales cuando examinamos la valoración que hacen de dicho proceso de inserción? El análisis realizado a nivel micro pone de relieve que tener un título universitario no implica otorgar la misma valoración a la educación recibida y cómo ésta es acogida en el mercado. Desde la perspectiva de la equidad, los diferentes indicadores subjetivos analizados muestran diferencias significativas. Estas no se deben especialmente al nivel formativo familiar. Sí, en cambio, son más significativas las diferencias según el género y el rendimiento académico. Ser mujer, tener buenas notas y haber estudiado un grado que no sea del área de Humanidades contribuye a tener una percepción positiva sobre la transición laboral. No obstante, es preciso señalar que la estrecha relación establecida en los niveles educativos inferiores entre el origen social de las familias y los resultados educativos, no desaparece en niveles superiores, sino que adquiere nuevas formas. Por tanto, de alguna forma también la posición familiar en la estructura social está incidiendo de forma indirecta en la calidad de la inserción (Fachelli \& Navarro-Cendejas, 2015).

Otra cuestión es el posible efecto de la crisis económica, lo cual se hace evidente en el inferior nivel de empleabilidad de los titulados en el 2014, y la ligera recuperación que se observa para el 2017, tal y como ya han concluido otros trabajos previos (AQU, 2014, 2017; OCDE, 2017). Pero el título no sitúa en un mismo plano a todos los titulados. Las mujeres y los graduados con mejor expediente son los más satisfechos. El análisis por área disciplinar dibuja los estudiantes de Humanidades como los más insatisfechos. Atendiendo a la satisfacción con la retribución, es unánime la insatisfac- 
ción manifestada para todos los colectivos estudiados y la no mejora. Eso es un efecto derivado de la crisis económica, para el que no se dan señales de recuperación, y que ha afectado a todos los estratos sociales, incluidos los titulados universitarios (PinedaHerrero et al., 2016). No obstante, éste es un sector de población altamente satisfecho con el contenido del trabajo.

Respecto a la reforma universitaria, la introducción del aprendizaje por competencias parece haber acercado la universidad a las demandas que los jóvenes perciben desde el mercado de trabajo. Así lo apuntan los diferentes análisis sobre las competencias adquiridas por los titulados, y más en concreto el indicador sobre el déficit formativo, que mejora en la edición 2017. Ello informa en un sentido positivo de los deberes que tenía la universidad en términos de los objetivos de empleabilidad marcados desde el EEES (Martínez \& González, 2018). Debe señalarse que algunas de las competencias instrumentales (idiomas y TICS) necesitan ser reforzadas, planteándose así el papel de la universidad en su formación (Llanes et al., 2017). Aunque no hay que perder de vista que estas conclusiones conviven con otros resultados no tan esperanzadores en estudios que analizan la valoración de los empleadores (Pineda-Herrero et al., 2018).

Algunas consideraciones adicionales se derivan del análisis realizado sobre la competencia trabajo en equipo, esta ha sido la mejor valorada por los encuestados y coincide con que es una de las competencias transversales por excelencia en la formación de grado. Por un lado, a la luz de cómo han cambiado las valoraciones de los titulados en un sentido positivo de una edición a otra, se evidencian los esfuerzos de adecuación de la institución a la formación exigible por parte de los empleadores. Por otro lado, la tendencia de las valoraciones a homogeneizarse en puntuaciones más altas refleja que existan diferentes velocidades de transformación y adaptación en las titulaciones. Ello puede responder a diferencias en las dimensiones organizativas, la cultura docente, y la disponibilidad de recursos, entre otros factores. Por tanto, en todas las enseñanzas se ha iniciado un proceso de transformación y modernización, pero con ritmos muy distintos.

Finalmente, un análisis sobre el nivel de ajuste de los titulados en el mercado de trabajo, apunta que existe un mayor porcentaje de estudiantes inseridos en el mercado laboral de acuerdo con su titulación, que aquellos que realizan otras funciones universitarias diferentes. Es difícil aún determinar unas tendencias claras de cambio comparando las dos ediciones; será necesario incorporar los resultados de nuevos titulados para alcanzar conclusiones definitivas. No obstante, en la mejora que se ha observado entre el ajuste de las competencias dadas en la universidad y utilizadas en el puesto de trabajo para la mayoría de titulados, se evidencia una mayor empleabilidad de estos jóvenes en el actual mercado de trabajo, caracterizado por su flexibilidad y permeabilidad. Asimismo, estos datos, también hacen pensar la misión de la universidad, transversalidad versus formación específica (Llanes et al., 2017; Silva, et al., 2014).

Hay que continuar estudiando la trayectoria profesional de los egresados más allá de los tres primeros años para conocer su cristalización en el mercado de trabajo y conocer si la equidad en la inserción se prolonga más allá de los primeros momentos. Así como incidir en análisis propios, al trabajar con datos secundarios algunas cuestiones de la inserción (bienestar, estado emocional, ...) no se han podido analizar ya que la encuesta no recogía estas dimensiones. En futuras investigaciones sería interesante 
incorporar los nuevos elementos de los indicadores subjetivos que recoge la literatura para seguir acercarnos al fenómeno de la inserción desde diferentes perspectivas. Otra línea de trabajo derivada del estudio sería abordar los indicadores subjetivos con una metodología cualitativa, lo cual nos llevaría a comprender los posibles mecanismos que operan en las cuestiones de percepción, y valoración que configuran las estrategias de inserción para las mujeres y aquellos jóvenes con mejor rendimiento. Los datos derivados del estudio cualitativo van a permitir mejorar el diseño de las políticas de orientación y dispositivos de asesoramiento previos a la elección de estudios, durante $\mathrm{y}$ al final de los mismos.

Agradecemos al Observatorio del Estudiante de la Universidad de Barcelona su apoyo para la realización de este estudio y a la Agència per a la Qualitat del Sistema Universitari de Catalunya por la cesión de la base de datos de los estudios de inserción de los titulados por el sistema universitario catalán.

\section{Referencias bibliográficas}

AQU CATALUNYA (2014). Universitat i treball a Catalunya 2014. Estudi de lainserció laboral de la población titulada de les universitats catalanes Recuperado de https://n9.cl/y3rm

AQU CATALUNYA (2017). La inserció laboral dels doctors $i$ doctores de les universitats catalanes. Recuperado de https://n9.cl/b4d7

Ariño, A. (2009). La dimensión social y la innovación en el Espacio Europeo de Educación Superior. Revista d'Innovació Educativa, 2, 2-9.

Berlanga, V., Figuera, P., \& Pons-Fanals, E. (2013). Incidencia de la beca salario: impacto, perfil y rendimiento de los estudiantes. RELIEVE, 19(2), 1-15. http://dx.doi. org/10.7203/relieve.19.2.2654

Bologna (1999). Declaración de Bolonia. Recuperado de https://n9.cl/eerp

Boudon, R. (1974). Education, opportunity and social inequality: changing prospects in Western society. New York: Wiley.

Bourdieu, P., \& Passeron, J. (1990). Reproduction in education, society and culture. Londres: Administrative and Social Studies Teesi de Polytechnic.

Camelli, A. (2006). An overiew of graduate employment. Bologna: Almalaurea.

Corominas, E., Villar, E., Saurina, C., \& Fàbregas, M. (2012). Construcción de un índice de Calidad Ocupacional (ICO) para el análisis de la inserción profesional de los graduados universitarios. Revista de Educación, 357, 351-374. http://dx.doi.org/104438/1988-592X-RE-2010-357-064

Comisión Europea (2010). EUROPA 2020. Una estrategia para un crecimiento inteligente, sostenible e integrador. Recuperado de https://n9.cl/dq2wn

Costa, L.V.,\& Duarte, J.S. (2011). Avaliação da Carreira no Mundo Contemporâneo: Proposta de um Modelo de Três Dimensões. Revista de Carreiras e Pessoas, 1(1), 1-22. https://doi.org/10.20503/recape.v1i1.6493

Delaney, A. (2008). Typical institutional research studies on students: Perspective and examples. New Directions for Higher Education, 141, 57-67.https://doi.org/10.1002/ he. 293

Erikson, R., \& Jonsson, J.O. (1996). Can education be equalized? The Swedish case in comparative perspective. Boulder: Westview. 
Escobar, M. (1998). Las aplicaciones del análisis de segmentación: el procedimiento Chaid. Empiria. Revista de Metodología de Ciencias Sociales, 1, 13-49. https://doi. org/10.5944/empiria.1.1998.706

Fachelli, S., \& Planas, J. (2014). Inserción profesional de los universitarios: de la expansión a la crisis. Revista Española de Sociología, 21, 69-98.

Fachelli, S., \& Navarro-Cendejas, J. (2015). Relación entre origen social e inserción laboral de los graduados universitarios. RELIEVE, 21(2), 1-22. http://dx.doi.org/10.7203/ relieve.21.2.7812

Figuera, P. (1996). La inserción socio-profesional del universitario/a. Barcelona: Ediciones UB.

Formichella, M.M., \& London, S. (2013). Empleabilidad, educación y equidad social. Revista de estudios sociales, (47), 79-91. https://doi.org/10.7440/res47.2013.06

González, C., \& Martínez, P. (2016). Expectativas de futuro laboral del universitario de hoy: un estudio internacional. Revista de Investigación Educativa, 34(1), 167-183. http://dx.doi.org/10.6018/rie.34.1.232071

Herranz, C., \& Muelas, D. (2019). Lo más leído en UNISÍ. La incorporación de los estudiantes universitarios al mercado laboral: fortalezas y dificultades [Mensaje de un blog]. Recuperado de https://n9.cl/tgvb

Lent, R.W., \& Brown, S.D. (2006). Integrating person and situation perspectives on work satisfaction: A social-cognitive view. Journal of Vocational Behavior, 69, 236-247. http://dx.doi.org/10.1016/j.jvb.2006.02.006

López, M.C., León, M.J., \& Pérez, P. (2018). El enfoque por competencias en el contexto universitario español. La visión del profesorado. Revista de Investigación Educativa, 36(2), 529-545.http://dx.doi.org/10.6018/rie.36.2.314351

Llanes, J., Figuera, P., \& Torrado, M. (2017). Desarrollo de la empleabilidad y gestión personal de la carrera de graduados en pedagogía. Revista Española de Orientación y Psicopedagogía, 28(2), 46-60. https://dx.doi.org/10.5944/reop.vol.28.num.2.2017.20118

Mañé-Vernet, F., \& Miravet-Arnau, D. (2007). L'adequació a la formació universitària. En A. Ramoneda (Ed.), Educació superior i treball a Catalunya: anàlisi dels factors d'inserció laboral(pp. 155-186). Barcelona: AQU.

Martínez García, J.S. (2017). Sobrecualificación de los titulados universitarios y movilidad social. Papers. Revista de Sociologia, 102(1), 29-52. http://dx.doi.org/10.5565/rev/ papers. 2225

Martínez, P., \& González, C. (2018). Orientación, empleabilidad e inserción laboral en la universidad a través de un Modelo de Ecuaciones Estructurales. Revista Española de Pedagogía, 76(269), 119-139. http://dx.doi.org/10.22550/REP76-1-2018-06

Martínez, P., González, C., \& Rebollo, N. (2019). Competencias para la empleabilidad: un modelo de ecuaciones estructurales en la Facultad de Educación. Revista de Investigación Educativa, 37(1), 57-73. http://dx.doi.org/10.6018/rie.37.1.343891

Obschonka, M. (2014). Enterpreneurship as 21st Century Skill: Taking a Developmental Perspective. En M. Coetzee (Ed.), Psycho-social CareerMetacapacities (pp. 307-320). London: Springer.

OCDE, (2017). Panorama de la educación. Indicadores de la OCDE 2017. Informe español. Recuperado dehttps://n9.cl/2ezh

Pineda-Herrero, P., Agud-Morell, I., \& Ciraso-Calí, A. (2016). Factores que intervienen en la inserción laboral de los titulados en Educación en tiempos de crisis: un estudio 
sobre Cataluña. Revista de Educación, 372, 141-169. http://dx.doi.org/10.4438/1988592X-RE-2015-372-318

Pineda-Herrero, P., Ciraso-Cali, A., \& Armijos-Yambay, M. (2018). Competencias para la empleabilidad de los titulados en Pedagogía, Psicología y Psicopedagogía: un estudio comparativo entre empleadores y titulados. Revista Española de Pedagogía, 76 (270), 313-333. http://dx.doi.org/10.22550/REP76-2- 2018-06

Sarramona, J., \& Santiuste, V. (2015). La formación no presencial ante el reto de las competencias profesionales. Revista Española de Pedagogía, 73(262), 449-464.

Silva, A.D., Taveira, M., Bernardo, E., \& Rodríguez, M.L. (2014). Perfiles de carrera en educación superior: un estudio en la Universidad Pedagógica de Mozambique. Revista de Investigación Educativa, 32(1), 25-39. http://dx.doi.org/10.6018/rie.32.1.161311

Suárez, B. (2016). Empleabilidad: análisis del concepto. Revista de Investigación en Educación, 14(1), 67-84.

Torrents, D., \& Fachelli, S. (2015). El efecto del origen social con el paso del tiempo: la inserción laboral de los graduados universitarios españoles durante la democracia. Revista Complutense de Educación, 26(2), 331-349. https://doi.org/10.5209/ rev_RCED.2015.v26.n2.43070

Troiano, H., Torrents, D., Sánchez-Gelabert, A., \& Daza, L. (2017). Evolución del acceso a la universidad y de la elección de titulación universitaria entre la población joven de Catalunya. Cuadernos de Relaciones Laborales, 35(2), 281-303. http//doi.org/10.5209/ CRLA.56775

Villarroya, R., \& Ramos-Villagrasa, P. (2017). Competencias clave para la empleabilidad de los egresados universitarios: Un análisis en la Universidad de Zaragoza. Acciones e investigaciones sociales, (37), 215-235. https://doi.org/10.26754/ojs_ais/ais.2017372193

Yorke, M. (2006). Learning and employability. Employability in Higher Education: what it is-what it is not. New York: Higher Education Academy.

Fecha de recepción: 3 de septiembre de 2019.

Fecha de revisión: 3 de octubre de 2019.

Fecha de aceptación: 29 de enero de 2020. 
岂
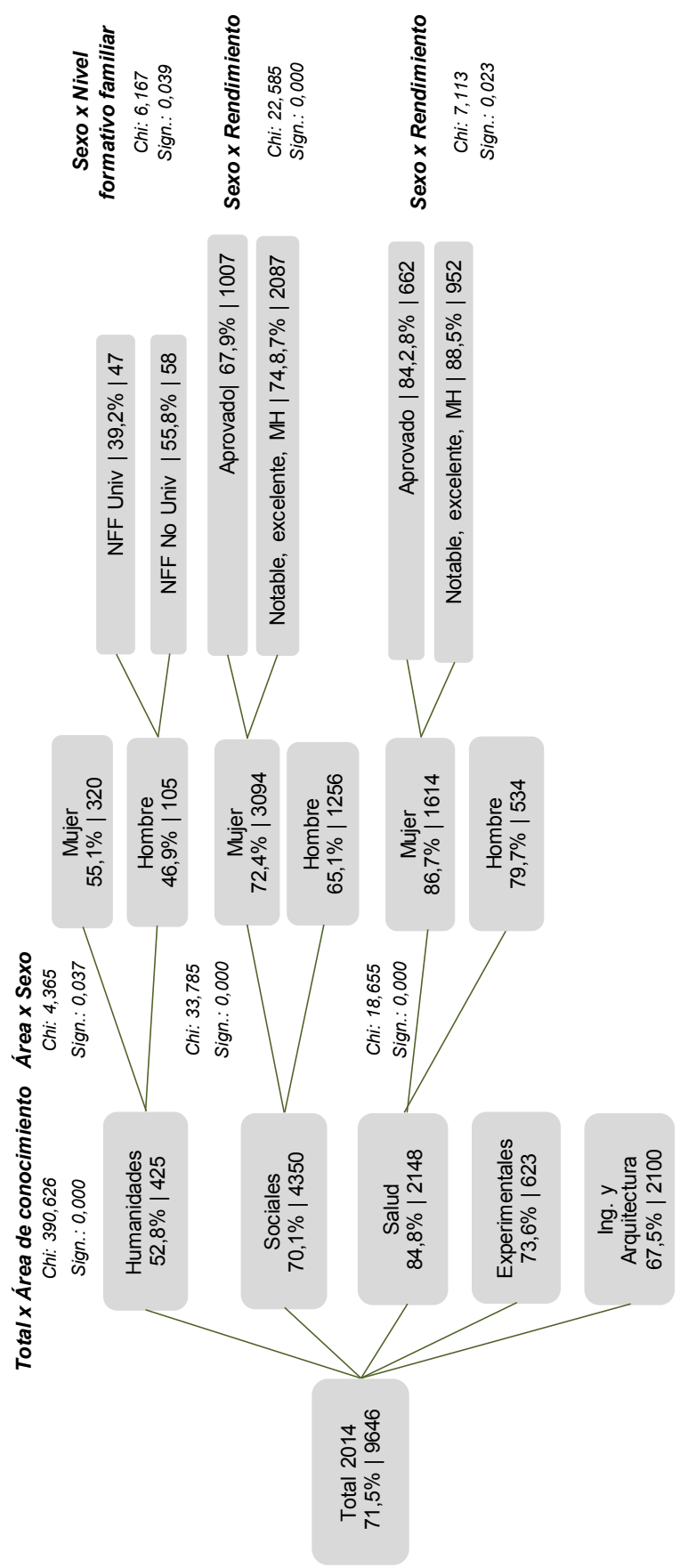

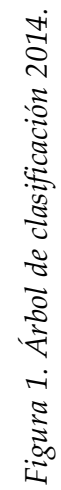




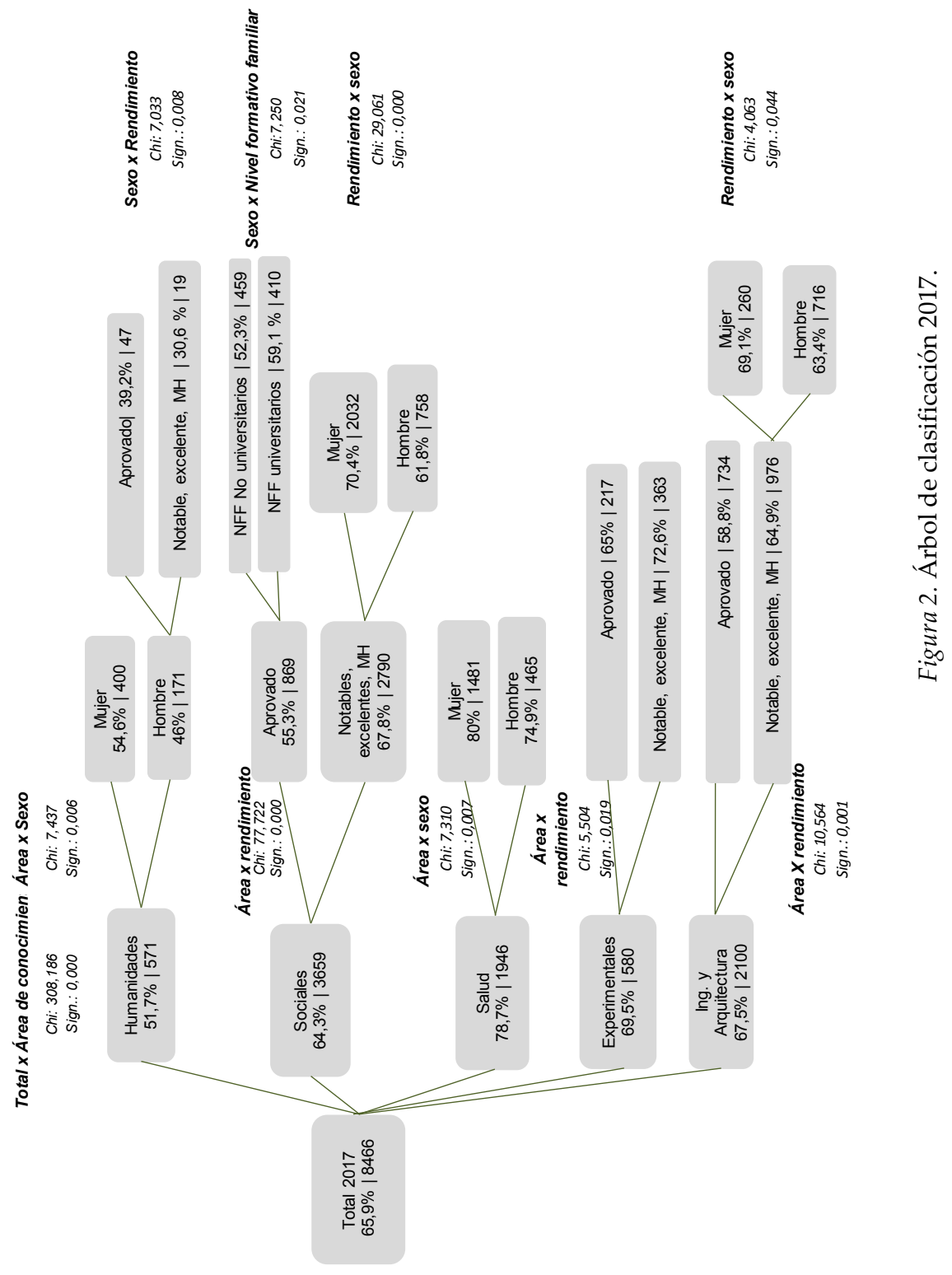

